\title{
Anaerobic two stage degradation pattern of fermentable components of municipal solid waste
}

\author{
Chanakya H.N. ${ }^{1,2, *}$ and Shwetmala K. ${ }^{1}$ \\ ${ }^{1}$ Center for Sustainable Technologies, IISc, Bangalore 560012 \\ ${ }^{2}$ Centre for infrastructure, urban planning and sustainable transport, IISc, Bangalore 560012. India \\ Received: 06/06/2017, Accepted: 03/12/2017, Available online: 09/01/2018 \\ *to whom all correspondence should be addressed: e-mail: chanakya@astra.iisc.ernet.in, shwetmala@gmail.com
}

\begin{abstract}
With increased contribution of household and kitchen wastes in overall MSW and its preferred conversion by biomethanation there is a need to study decomposition rates and kinetics, 'leachate potential' and related issuesespecially to promote decentralized treatment. Anaerobic digestion characteristics of ten predominant types of the fermentable components (fruits, vegetables and their mixture) commonly found in urban solid waste in Bangalore were examined under lab conditions by monitoring biogas production in micro-digesters at room temperatures $\left(25 \pm 3^{\circ} \mathrm{C}\right)$. The rate of decomposition, the pattern of fit and extent and rate of biogas production from these potential feedstocks are analyzed to understand the degradation kinetics. All feedstocks showed a two stage linear fit for gas production comprising of a rapid initial phase lasting 10-20days accounting for $>70 \%$ gas production followed by a slower second phase. Degradation could be predicted using a two stage linear fit transiting from initial to a second stage at an appropriate unique transition point, yet similar amongst fruits or vegetables. The need for large aceticlastic capability (rapid initial degradation) thus becomes a criteria for biomethanation process of such feedstocks.
\end{abstract}

Keywords: Fermentables, biological methane potential, biogas, Two-stage decomposition fit

\section{Introduction}

Resource recovery and processing of organic fraction of municipal solid wastes (OFMSW) has been receiving considerable attention today primarily due to the increasing organic fraction in the municipal solid waste (MSW) generated in most of the Indian cities (NEERI 2005; Pandey et al., 2007; Chattopadhyay et al., 2009; Kumar and Goel, 2009; Chanakya and Swami, 2011). As a consequence, the associated aesthetic and environmental problems necessitate alternative disposal methods. For example, Bangalore city is one such metro in India which not only produces a lot of MSW (>3500 tons per day, tpd; Chanakya and Sharatchandra, 2005; Chanakya et al., 2009a; 2009b), but also the decomposable organic fraction has risen to high levels (>75\%). In freshly collected MSW from households about $72-93 \%$ was the biodegradable organic wastes in most towns and cities of Karnataka (Chanakya and Swamy, 2011). In Bangalore city, it has been observed that $>90 \%$ of the generated 3500tpd is collected and generally reaches various authorized and unauthorized waste disposal sites around the city (Chanakya et al., 2011; Chanakya et al., 2015). Of the collected MSW about $10 \%$ has been estimated to reach unauthorized and often ephemeral dumps (within and outside the city). Such temporary dumps or collection points provide a stop-gap arrangement for storing MSW for short duration and transporting it away when convenient. Obviously, this approach is aesthetically and environmentally unsatisfactory (Shwetmala et al., 2012) and often causes huge nutrient flows that can be much larger than the capacity of the local ecosystem to handle through natural processes (c.700kg N/ha/yr, Chanakya and Sharatchandra 2005). Therefore, in the presence of such ephemeral storage or random open dumps, there is a likelihood of a rapid anaerobic digestion and a predisposition to produce many GHGs predominantly methane. In order to overcome such tendencies, a decentralized approach to treatment of the organic fraction as well as the decentralized processing of recyclables has been suggested to be potentially economic, aesthetic and sustainable (Dhindaw et al., 2014). Considering that MSW contains predominantly fermentable wastes of domestic origin, there is a need to identify potential options for decentralized processing of such fermentable wastes (OFMSW; Cecchi et al., 1997; Mata-Alvarez et al., 2000). Possible decentralized treatment methods for OFMSW under these conditions are aerobic composting and anaerobic degradation (biomethanation). With such an organic-rich composition and when stored in the open, rapidly biodegradable components OFMSW such as fruits, vegetable and food wastes become subject to rapid degradation. The resulting accumulating products are generally volatile fatty acids (VFA) and other odoriferous intermediate compounds. The leaf litter component of MSW however decomposes a lot more slowly and only rarely leads to strong mal-odours (Chanakya et al., 2009a).

VFA intermediates that emerge in leachate from MSW degradation may be both aesthetically and 
environmentally unacceptable even in small dumps and temporary storage sites. Therefore, an understanding of the propensity to rapidly decompose and produce odouriferous leachate is of importance to evolve safer and acceptable alternatives. Leachate is produced under both aerobic and anaerobic conditions (Barlaz et al., 1989; Canet and Pomares, 1995; Hamoda et al., 1998; Monedero et al., 1999; Chanakya et al., 2007a; b). Under anaerobic digestion, rapid VFA production is known to inhibit the second step of anaerobic digestion namely methanogenesis. The decomposition of typical mixtures of vegetable, fruit and garden wastes (as in MSW) has been studied and the potential of rapidly degrading food wastes components to produce VFA and lower reactor $\mathrm{pH}$ has also been reported (Chanakya et al., 2009b). However, only a few of such substrates were studied earlier (Gunaseelan, 2004; Chanakya et al., 2009b). Therefore, an investigation that determines the rate of initial and subsequent decomposition needs to be carried out for a larger basket of common feedstocks in order to design and operate anaerobic digesters (Ravikumar, 2014).

During biomethanation, unless the two dominant steps of hydrolysis (leading to VFA production) and methanogenesis are matched carefully, hydrolytic microflora generally tends to overproduce VFA intermediates and quickly accumulates VFA such as acetic, propionic and butyric acids. These in turn suppress the methanogenic stage of the biogas production process. In such cases, the methanogenic microflora is soon outgrown by 'feedstock hydrolysing bacteria' and this situation results in a nearly irreversible accumulation of VFA (termed souring, Hill and Bolte 1989). However, under conditions of stable anaerobic digestion process all VFA produced is rapidly converted to methane and carbon dioxide (biogas) leading to a steady state process (Chanakya et al., 1999). MSW in Bangalore and most cities in India contain many rapidly degradable feedstock constituents that can make decentralized anaerobic treatment somewhat problematic. When MSW is allowed to accumulate VFA and highly "sour" MSW is fed to biogas plants or composting units, further decomposition is usually arrested. It thus becomes important to firstly determine rate of waste biodegradation and from this generate a usable index or measure in order to estimate the rate of decomposition and determine the rapid hydrolysis rates to serve as an index for process design. It is best to determine process kinetics using experimental data obtained under appropriately controlled conditions.

The physical nature and the chemical composition of organic wastes have long been considered as critical factors in determining the extent and rate of decay (Singh and Gupta, 1977). The content of pectin, cellulose, hemicellulose and lignin and the $\mathrm{C} / \mathrm{N}$ ratio are important controlling factors in the rate of anaerobic decomposition (Chanakya et al., 1999; 2009b; Ravikumar, 2014). All these above fractions of MSW do not degrade at the same rate; especially when relative content of simple polysaccharide, pectin, cellulose, hemicellulose and lignin vary from one substrate to another. As the composition of fruits, vegetables and food wastes vary significantly across different regions of the world, it is important to characterize the wastes for these constituents and assess their influence on the rapid decomposition phase.

Current understanding suggests that there are typically two classes of constituents in various waste components the hot water extractables and the more recalcitrant remaining matter. Sometimes it is also classified into those simpler to degrade constituents (such as hemicellulose -as extracted by $0.5 \mathrm{M}$ acid) and the remaining ligno-cellulosic components (digestible by cold $72 \% \quad \mathrm{H}_{2} \mathrm{SO}_{4}$ and undigestible residue - lignin). As a result of physical entrapment of degradable components (predominantly cellulose) by lignin, the extent of anaerobic biodegradability and the rate of biodegradation of various constituents of biomass feedstocks vary. In this study an attempt has been made to determine the rate and extent of degradation while attempting to separate the rapid hydrolysis stage from the slower ligno-cellulosic break down stage. The slope of the product formed, namely the cumulative biogas produced under standard test conditions (biological methane potential, BMP, assay) is visualized to have two rate components. A rapid initial phase is a result of the breakdown of simple compounds (water extractables or simply referred to as extractables, Ravikumar, 2014) that soon tapers off to yield a lower rate of gas production in the next stage. This slowing of gas production is assumed to emerge predominantly from the degradation of the less accessible ligno-cellulosics. The slope then may be considered to occur as two degradation rates distinctly transiting from the first to the second stage. The first rapid degradation segment (from extractables) is expected to have a 'rapid' rate constant while the latter arising from slow to degrade components (lingo-cellulosics) is expected to exhibit a lower decay constant or gas production rate with undigestible residue of lignin. While a few attempts have been made to model decay constants of OFMSW containing low extractables $(<10 \%)$, only a few of such attempts have been made in experimental studies of degradation rates where the overall gas production plot has been being split into a rapid early decay followed by a slower second stage of digestion with a hazy (or gradual and often not so distinct) point of transition (Chanakya et al., 2009a, b; Ravikumar, 2014). A clear distinction would be required for assessing degradation of feedstock components emerging from either extractables and lignocellulosics separately. From many of the earlier studies indicated above we attempt to implicate the early biogas production micro-digesters to be arising from anaerobic digestion (acidogenesis methanogenesis) from extractables and the slower second stage decomposition emerging from degradation of lingo-cellulose. Microdigesters used here indicate ideal anaerobic conditions of acidogenesis-methanogenesis and therefore indicate 'uninhibited rates under fermentation conditions employed.

We pursue the second approach to obtain greater insights and possibly, accuracy of prediction. Therefore, in this study a two phase kinetic pattern is visualized to measure 
the rate of anaerobic decomposition of ten specific types of fermentable components of MSW commonly occurring in urban solid waste of Bangalore and its potential to predict assessed. The rate kinetics of decomposition of the easy to degrade materials were estimated and compared at two different concentrations of feedstock $(0.5 \%$ and $0.25 \% \mathrm{TS}$, conditions that avoid accumulation of VFA $>5 \mathrm{~g} / \mathrm{L}$, Chanakya et al., 1999). These biodegradation rate constants at very low feedstock concentrations firstly indicate the highest rates of hydrolysis and these are expected to provide greater insights for designing reactors and predicting gas production and reactor health under ideal conditions of an un-inhibited nature and sufficiency of methanogenic microbial inoculum.

\section{Materials and methods}

\subsection{Feedstock selection}

Ten specific biomass wastes representing a wider basket of fermentable components or fractions of MSW of Bangalore was collected from IISc campus in Bangalore, India. We selected five vegetable wastes which included cauliflower (Brassica $s p$ ), onion (Allium $s p$ ) peels, flat beans (Vicia $s p$ ), radish (Raphanus $s p$ ) and peas (Pisum $s p$ ) and five fruits waste which included banana (Musa $s p$ ) peel, "Mousambi"/ sweet lime (Citrus sp.) peel, orange (Citrus $s p$ ) peels, papaya (Carica $s p$ ) and watermelon (Citrullus $s p$ ) peels (dominant for the season). Each of these ten individual components was collected fresh from source (within 12 hours of generation). The decomposition of a few leafy biomass feedstocks (Chanakya et al., 1999), cauliflower and fruits in MSW, banana and orange peel (Chanakya et al., 2009a; b) have been reported earlier and hence were used as internal checks. In addition, for this study, we chose onion, flat beans, radish, peas, sweet lime, papaya and watermelon for which there were no anaerobic degradation data. To make representative samples of mixed fruits, mixed vegetables and mixed fruits and vegetables, a mixture of equal proportions of mixed vegetable wastes, mixed fruit peels and wastes as well as mixture of fruits and vegetables, respectively, were subject to anaerobic degradation by BMP assay. In this way a total of ten individual feedstocks (separately) and three mixed feedstocks were studied.

\subsection{TS, VS and subcomponents estimation of feedstocks}

Freshly collected feedstocks were cut to $20-30 \mathrm{~mm}$ pieces. The total solids (TS) and volatile solids (VS) content of individual samples were determined from fresh samples using standard methods (APHA 1975). The TS was determined by drying wastes samples at $90 \pm 2{ }^{\circ} \mathrm{C}$ till constant weight (to avoid cellulose breakdown). One gram of dried powdered sample was placed in pre-weighed porcelain crucible and placed in the muffle furnace at $550 \pm 5{ }^{\circ} \mathrm{C}$ for $3 \mathrm{hrs}$. Samples were allowed to cool in a desicator and weighed after reaching room temperature. The percent weight loss on ignition gave the total volatile solids, a surrogate for organic matter content of the feedstock.
The subcomponents or structural components of each dried powdered individual feedstock were also determined. Three replicates (1g) were used in this analysis involving sequential extraction for various subcomponents (Chesson, 1978, ethanol extraction not done). By this assay these feedstock samples were fractionated into six fractions namely: i) hot water extractable pectin and sugars; ii) ammonium oxalate soluble pectin; iii) dilute acid $\left(0.5 \mathrm{M} \mathrm{H}_{2} \mathrm{SO}_{4}\right)$ extracted hemi-cellulose; iv) $72 \%$ acid extracted cellulose; $v$ ) residual lignin; and vi) ash remaining after combustion in the lignin fraction. Two modifications to this protocol (Chesson, 1978) were adopted. The material remaining after extraction with $72 \%$ sulphuric acid was further extracted from the sintered glass crucible after weighing. The extracted mass (representing lignin) was weighed once again and was placed in a muffle furnace at $600{ }^{\circ} \mathrm{C}$ for $1 \mathrm{hr}$ to ensure complete combustion of lignin. Further, with these samples, the first step of ether extraction for fats and lipids was not performed as they were expected to have low lipid content based on previous experience and also likely proteins extracted in each fraction was ignored due to their low concentrations.

\subsection{Biological methane potential (BMP) assay}

BMP assay (Owen et al., 1979; Owens and Chynoweth, 1993; Chanakya et al., 2007a) is useful to determine both the gas potential as well as the rate of anaerobic degradation of feedstocks to methane (Fig. 1). BMP was determined by fermenting powdered dry individual feedstock and their typical mixtures of equal proportion (vegetables, fruits and vegetables-fruits) with acclimatized inoculum at reasonably high levels. This test was carried out with $50 \mathrm{ml}$ of inoculum+substrate mixture placed in 135 $\mathrm{ml}$ serum vials with $0.5 \%$ and $0.25 \%$ TS and monitored for 60 days (Chanakya et al., 2007a). Inoculum was collected from a working biogas digester enriched with methanogens. Aceticlastic methanogens were enriched in the inoculum by adding $1 \mathrm{~g} / \mathrm{L}$ equivalent of acetate daily for two weeks. The $\mathrm{pH}$ of the inoculum was monitored daily for 14 days until pH stabilized between 7.5 and 8.5 indicating high aceticlastic activity and acetate utilization. No acetate was fed for next 7 days. This inoculum was filtered through fine cloth and used within 2 days. This ensured that aceticlastic methanogens were present in high numbers and prevented possible 'souring' of micro-digesters.

After addition of enriched methanogenic inoculum to the BMP vials, the air in the headspace was immediately displaced by flushing rapidly with biogas from a biomass fed biogas plant and the bottles were capped with rubber stoppers immediately and crimped with an aluminium seals within 1-2 min thereafter. Next, the headspace was flushed with oxygen free nitrogen to remove all traces of methane and the vials were incubated upside down for $\mathrm{BMP}$ assay. These BMP serum vials were incubated at room temperatures $\left(25 \pm 3^{\circ} \mathrm{C}\right)$.

The gas production in these vials was recorded by downward displacement of water at pre-determined. For reasons of convenience as well as from previous experience, the gas production in vials containing 
feedstock mixtures were measured at intervals of 1, 2, 4, 9, $14,26,34$ and 49 days after start so as to provide easily measurable gas volumes that remained less than the full volume of the burette used $(50 \mathrm{~mL})$. Experiments for BMP assay were run until cumulative gas generation reached a near plateau ( $<10 \%$ change in cumulative gas in last $20 \mathrm{~d}$ ). Inoculum-free controls were included in the BMP assay to estimate the gas production evolved from feedstock without the addition of inoculum. All BMP assays were carried out in triplicate (except for feedstock mixtures where one replicate was used for incubation). The cumulative gas production was plotted to determine the rate and extent of gas production and expressed as $\mathrm{ml}$ biogas per gram TS added. The head space gas composition was determined when the gas production rate appeared to reach a stationary phase or became low for all the vials. Gas analysis was carried out using a gas chromatograph equipped with a Porapak-Q column connected to thermal conductivity detector.
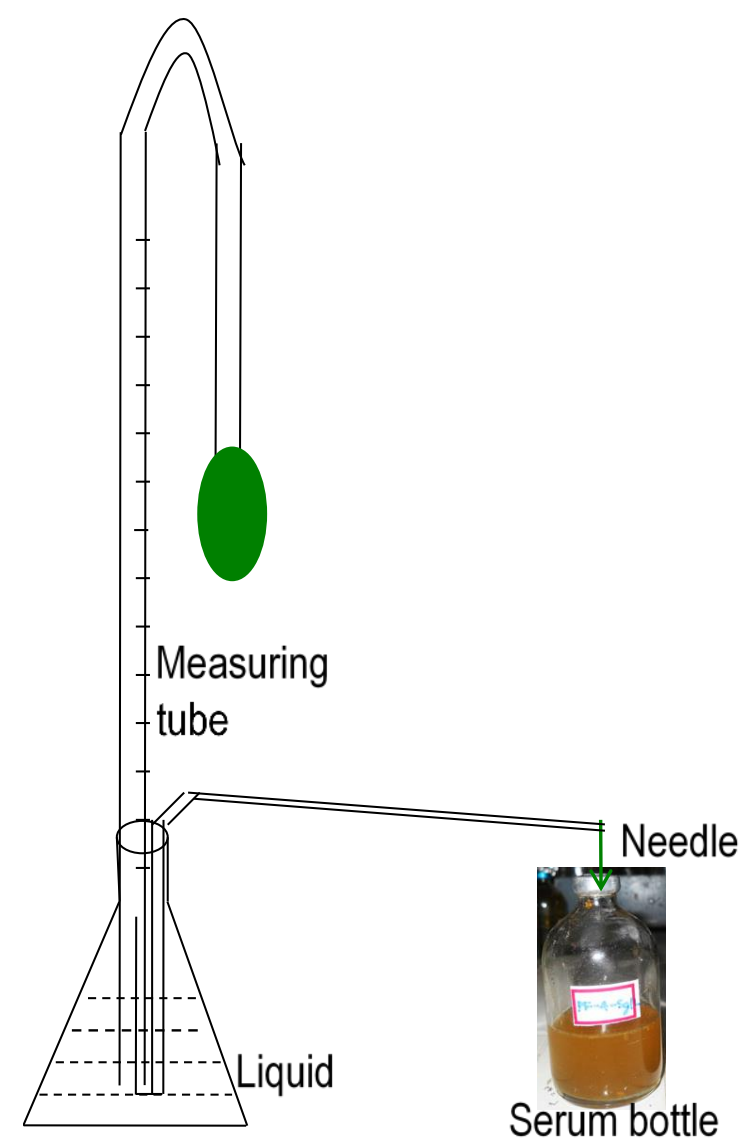

Figure 1. Schematic representation of experimental setup

From the plots of cumulative gas production two separate near-linear zones were selected for each of the substrates. The first linear segment of the plot was expected to represent the rapid gas production phase that is believed to be a result of decomposition predominantly from the water extractives (easy to degrade components). The slope and best linear fit of this was plotted in a standard spread sheet software. A similar effort was repeated for the near linear segment of the second low gas production stage. This second slope was expected to emerge from the decomposition of the difficult to degrade cellulose and ligno-cellulosics. The point where the two best fit plots appear to intersect was considered the point of transition in the gas production between the rapid gas production phase to the slow gas production phase. In a few cases the gas production did not start from the day of start. In such cases where the first best fit plot of the first slope crossed the $x$-axis, it was considered to be the acclimation period. Thus, the day representing the start and the end of the rapid gas production phase, the days representing the start and end of the slow second gas production stage and the point of transition was captured. It is expected that this data would represent the kinetics under ideal conditions i.e. not subject to substrate, end-product or methanogen inhibition. Under field conditions where situation is less than ideal, the degradation and gas production rates are expected to occur at levels lower than these rates.

\section{Results and discussion}

\subsection{Composition of waste substrate}

All components of MSW were collected fresh in order to simulate a situation that would occur during decentralized MSW treatment systems and would be representative of freshly collected MSW. These individual components of MSW are found at various levels of moisture content and therefore the TS and VS information become important. The TS content of different components ranged from 14 to $29 \%$ except for onion peels ( $>90 \%)$ and watermelon $(<10 \%$; Table 1) and occurred in the expected range. Onion, flat beans, peas, sweet lime and orange have a high concentration of VS (>90\%). Therefore, these may be considered to contribute more to the overall biogas production or compost residue on an 'as received' basis. Cauliflower waste on the other had the highest ash content (18\%) indicating the presence of inorganic constituents and possibly a higher level of drying and not expected to contribute to biogas production. This level of minerals is common in typical leaf biomass and its occurrence in cauliflower is considered as normal.

Within each of these feedstocks, the constituents such as pectin, hemicellulose and cellulose are easily degradable compared to lignin. The lignin content of all the feedstocks studied was low and generally occurred at levels of $\leq 10 \%$. Among these, watermelon and cauliflower wastes had lignin contents in a somewhat high range (10\%), whereas papaya had lower lignin content (6\%). Orange, papaya, sweet lime, peas and flat beans have a higher percentage of pectin, hemicellulose and cellulose (degradable fraction, $\geq 85 \%$, Table 1). The lower content of lignin in all feedstocks generally portends a near complete degradation while the lower content of lignin could translate into a higher rate of degradation. Such a high content of fruit and vegetable wastes in the MSW represents an easy to decompose feedstock where decomposition rates can reach such a high level that there would be very little undegradable /undegraded residue left as compost. In the past, leaf litter from road sweepings and garden wastes represented 
between $15-25 \%$ of the freshly generated MSW (Rajabapaiah, 1988; TIDE, 2000). Today in the absence of a large component of leaf litter or garden wastes components (with higher lignin content 15-40\%, Berg and
McClaugherty, 2013) in MSW it is unlikely that appreciable levels of compost can be recovered from such components of MSW or MSW with predominantly kitchen wastes.

Table 1. Content of TS, VS, and structural constituents in selected waste substrate used in anaerobic degradation

\begin{tabular}{ccccccccc}
\hline Waste substrate & TS & VS & Ash & Pectin 1 & Pectin 2 & Hemicellulose & Cellulose & Lignin \\
\hline Cauliflower & $22 \%$ & $82 \%$ & $18 \%$ & $34 \%$ & $10 \%$ & $17 \%$ & $22 \%$ & $9 \%$ \\
Onion & $91 \%$ & $92 \%$ & $8 \%$ & $32 \%$ & $23 \%$ & $13 \%$ & $17 \%$ & $10 \%$ \\
Flat beans & $29 \%$ & $91 \%$ & $9 \%$ & $39 \%$ & $11 \%$ & $20 \%$ & $16 \%$ & $9 \%$ \\
Radish & $10 \%$ & $85 \%$ & $15 \%$ & $50 \%$ & $11 \%$ & $6 \%$ & $16 \%$ & $9 \%$ \\
Peas & $22 \%$ & $94 \%$ & $6 \%$ & $51 \%$ & $9 \%$ & $14 \%$ & $12 \%$ & $8 \%$ \\
Banana & $21 \%$ & $87 \%$ & $13 \%$ & $53 \%$ & $5 \%$ & $7 \%$ & $11 \%$ & $9 \%$ \\
Sweet lime & $27 \%$ & $94 \%$ & $6 \%$ & $40 \%$ & $22 \%$ & $13 \%$ & $12 \%$ & $9 \%$ \\
Orange & $28 \%$ & $95 \%$ & $5 \%$ & $55 \%$ & $16 \%$ & $9 \%$ & $10 \%$ & $8 \%$ \\
Papaya & $14 \%$ & $84 \%$ & $16 \%$ & $54 \%$ & $12 \%$ & $10 \%$ & $14 \%$ & $6 \%$ \\
Watermelon & $7 \%$ & $89 \%$ & $11 \%$ & $55 \%$ & $11 \%$ & $5 \%$ & $12 \%$ & $10 \%$ \\
\hline
\end{tabular}

Pectin-1 (hot water extractables) and pectin-2 (hot oxalate extractables) should be construed to contain some structural proteins and minerals and are not entirely pectins

\subsection{Biological methane production assay}

In this study BMP assay has been utilized as a measure of substrate decomposability under typical anaerobic (as well as biomethanation) conditions. This was carried out to determine the period for which rapid decomposition is likely to occur and to estimate the gas production potential from different components of MSW under near ideal conditions of TS, inoculum and physico-chemical conditions.

The result of BMP assay at 0.5 and $0.25 \%$ TS concentration of feedstock is presented in Figs 2-4 and 5-7, respectively. This level was chosen such that at any condition of rapid degradation the maximum VFA levels that could accumulate could not cross a threshold of $5 \mathrm{~g} / \mathrm{l}$ - a level which VFA has been found to be inhibitory to methanogens of this inoculum. Degradation of each of the fruits and vegetables under anaerobic degradation at $0.5 \%$ concentration are shown in Fig 2 and 3 and at $0.25 \%$ concentration are shown in Fig 5 and 6 . Typical mixtures of these vegetables and fruits at $0.5 \%$ concentration and at $0.25 \%$ concentration were fermented anaerobically to determine BMP and are presented in Figs 4 and 7. The biogas production in controls (not shown, only feedstock without inoculum) had always shown very low biogas production levels compared to samples with fruit and vegetable feed stocks (feedstock with inoculum). The cumulative biogas production in control generally reached a plateau within 2 to 10 days of initiation suggesting a typical VFA generated inhibition of methanogenesis or a stalled process due to the absence of adequate methanogenic population. This clearly shows that appropriate inoculum is important for biogas production as well as sustaining a high biogas production rate throughout.

The biogas production ranged from 215 to 605 and 227 to $610 \mathrm{ml} / \mathrm{g}$ of TS, at 0.5 and $0.25 \%$ TS, respectively. Citrus fruit rinds from orange and sweet lime showed low gas production levels as expected (Gunaseelan, 2004; Chanakya et al., 2009b). For citrus fruit peels two modes of inhibition are suspected. Firstly, being pectin rich, they have a tendency for rapid VFA formation which inhibits methanogenesis and second more important is the presence of various anti-microbial agents in the essential oils (Gunaseelan, 2004). Flat bean (Vicia $s p$ ), and onion (Allium sp.) showed a high gas production rates and total gas production whereas banana peel showed a moderate gas production level $(\approx 421 \mathrm{ml} / \mathrm{g}$ TS at $0.25 \% \mathrm{TS}$ and $\approx 468 \mathrm{ml} / \mathrm{g}$ TS at $0.5 \%$ TS). From available data it is not clear as to why banana peel with a high pectin content and potentially high degradability should have lower biogas production levels. A more lignified banana leaf, sheath and rachis produce similar biogas production levels.

This moderate gas production levels has occurred in the absence of a high lignin content and therefore need further research (Chanakya and Malayil, 2012). Mixed fruit, mixed vegetable and mixed fruit+vegetable had moderate gas production of 484,407 and $446 \mathrm{ml} / \mathrm{g} \mathrm{TS}$, respectively at $0.5 \% \mathrm{TS}$ and a near similar gas production level was found at $0.25 \% \mathrm{TS}(472,413$ and $446 \mathrm{ml} / \mathrm{g} \mathrm{TS}$, respectively). While individual components gave higher gas production levels and in the absence of any appreciable lignin content (as is found with other biomass), it is not clear from available data as to why gas production from the combined substrates is lower than the arithmetic average gas production level.

It is generally known that mixing a few substrates generally increases gas production levels above the average because of increased availability of nutrition and yet these mixtures show a lower level of biogas and therefore needs further research. In these mixed feedstocks, the gas composition measured have generally showed the presence of $>50 \%$ of methane. This indicates that the rate of acidogenesis and methanogenesis were well balanced. 

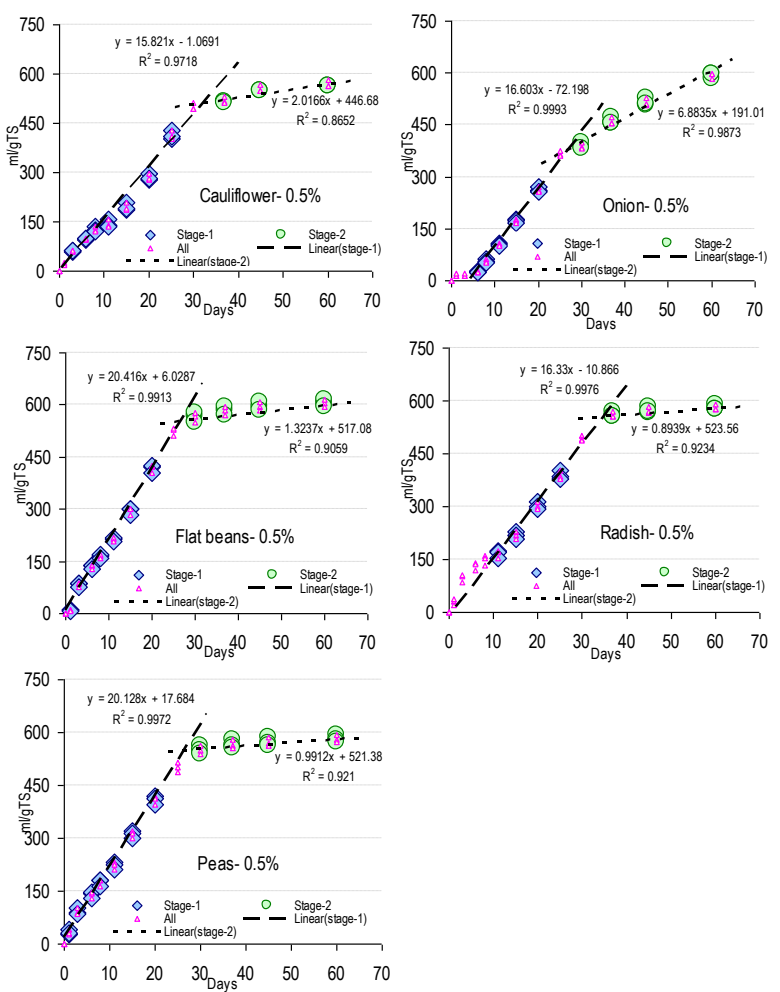

Figure 2. The cumulative gas production measured during various stages of BMP assay at $0.5 \%$ TS concentration in the fermenting mass of the commonly used five vegetable feedstocks (Cauliflower, Onion, Flat beans, Radish, Peas) during phase- I and II of degradation. The black dotted line shows best linear pattern after removing outliers.
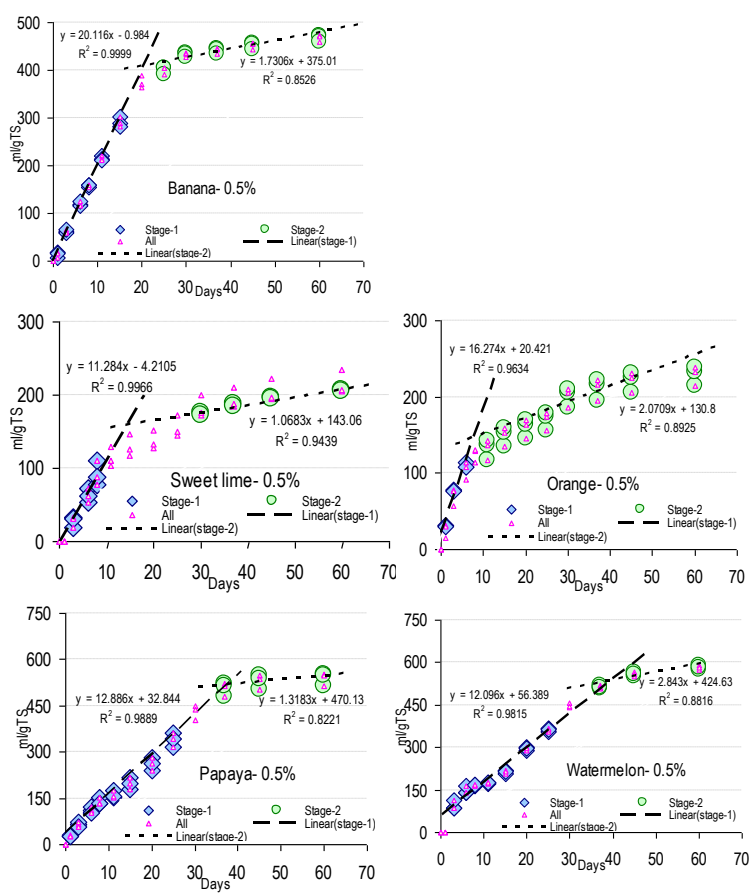

Figure 3. The cumulative gas production measured during various stages of BMP assay at $0.5 \%$ TS concentration in the fermenting mass of the commonly used five fruit

feedstocks (Banana, Sweet lime, Orange, Papaya,

Watermelon) during phase- I and II of degradation. The

black dotted line shows best linear pattern after removing outliers
Cauliflower, peas, watermelon and flat beans showed a good methane content (>60\%) and it is an indication of absence of a condition that represents VFA induced suppression of methanogenesis. Whereas at $0.5 \% \mathrm{TS}$, orange and sweet lime showed poor methane yield of $23 \%$ and $20 \%$, respectively. At $0.25 \%$ TS, sweet lime, mixed fruits and mixed fruit+vegetable showed a methane content of $24 \%, 29 \%$, and $44 \%$, respectively. This pattern of low methane generally occurs due to accumulation of VFA and subsequent methanogenic inhibition. There is thus a need to determine an acceptable level of citrus fruits that can be fermented as mixtures in the anaerobic digester without suffering an inhibition of the process. While a lot of previous research tends to assume the inhibition due to presence of essential oils and their microbial inhibition, current data seems to suggest the inhibition to be occurring due to the presence of a high content of pectin and its rapid break-down. It therefore requires a deeper investigation.

\subsection{Degradation pattern and rate kinetics}

The biogas evolution data revealed that anaerobic decomposition of various organic wastes generally occurred in two stages. The results obtained are shown in Figs 2, 3, 4 and 5, 6, 7. Among the feedstocks studied banana peel, orange peel and pea shells showed a clear two stage degradation at $0.5 \%$ TS. Water melon showed a two stage degradation pattern at $0.25 \%$ TS.
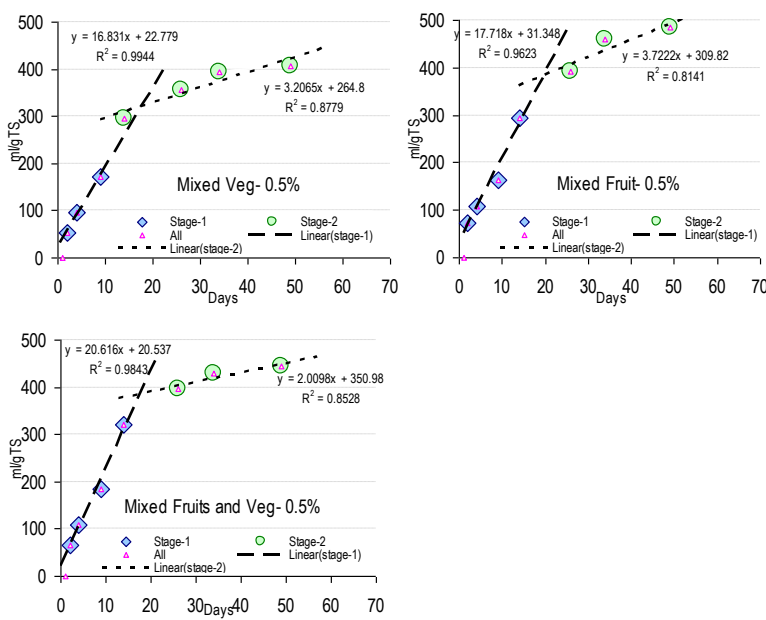

Figure 4. The cumulative gas production measured during various stages of BMP assay at $0.5 \%$ TS concentration in the fermenting mass of the equal proportionally mixture of vegetables (mixed veg), fruits (mixed fruit) and mixture of vegetables and fruits (mixed fruits and veg) during phase- I and II of degradation. The black dotted line shows best linear pattern after removing outliers.

The other feedstocks showed a somewhat different pattern. The extent of substrate degradation (as determined by gas evolved) in phase 1 is expected to emerge from the easily decomposable fractions of waste pectins 1 and 2, starches, etc., whereas the slower phase 2 represents breakdown of the slow to decompose and/or resistant fractions that is expected to include structural 
hemi-cellulose, cellulose and possibly some lignin (Chanakya et al., 1999; Chanakya and Malayil, 2012).

Among the ten feedstocks, banana peel, cauliflower leaves at $0.5 \% \mathrm{TS}$ and all feedstocks other than citrus peels had a rapid first phase that yielded over $75 \%$ of the cumulative gas yield. In these latter cases the phases 1 and 2 representing decomposition of extractables and structural materials were merged as stage-I. Usually the transition to stage-II occurred between 20 to 30 days of incubation at $0.5 \%$ TS whereas it occurred between 15 to 25 days of incubation at $0.25 \%$ TS with the exception of citrus fruit peel. Decomposition of sweet lime and orange peels showed an inhibited decomposition pattern and therefore needs to be excluded from the 2-stage pattern.

The pattern of cumulative biogas production has been separated into first and second stage degradation as described earlier. The pattern at $0.5 \%$ TS for vegetables, fruits and feedstock mixtures are presented in Figs. 2, 3 and 4 , respectively. Similarly, the pattern of cumulative biogas production data at $0.25 \%$ TS for vegetables, fruits and feedstock mixtures are presented in Fig. 5, 6 and 7.
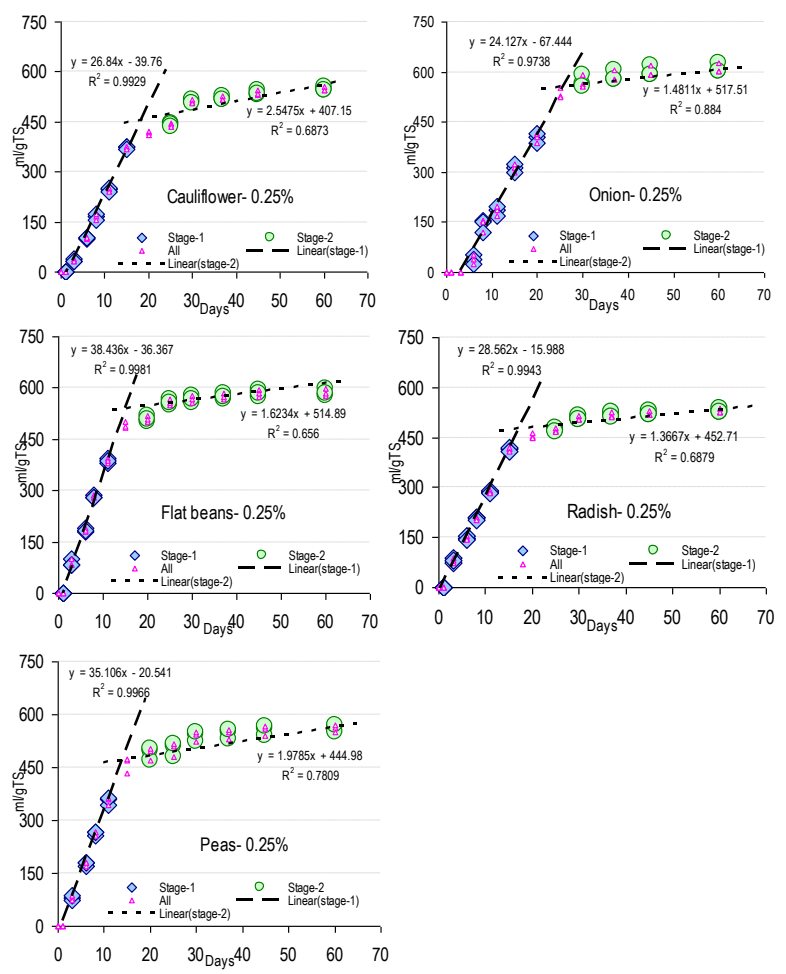

Figure 5. The cumulative gas production measured during various stages of BMP assay at $0.25 \%$ TS concentration in the fermenting mass of the commonly used five vegetable feedstock (Cauliflower, Onion, Flat beans, Radish, Peas) during phase- I and II of degradation. The black dotted line shows best linear pattern after removing outliers.

All feedstocks fermented at $0.5 \%$ and $0.25 \%$ TS concentration provided a reasonable fit to estimate degradation (gas production) for stages I and II separately. Among all feedstocks at $0.25 \%$ and $0.5 \%$ TS concentrations, phase-I biogas production (substrate conversion) accounted for a total gas production between $75-90 \%$ with the exception of citrus fruit peels. The initial phase was rapid compared to the latter phase-II. For example, cauliflower decomposed very rapidly producing about 70\% cumulative gas in 15 days at $0.25 \%$ TS concentration and $>70 \%$ gas are produced in 25 days of degradation at $0.5 \%$ TS concentration. Among most of the feedstocks $>70 \%$ of the total gas is produced before reaching the transition point after which the rate of decomposition begins to slow down in phase-II. In citrus fruits (sweet lime and orange) only about $40 \%$ of gas was produced in phase-I. More research is required to explain this.
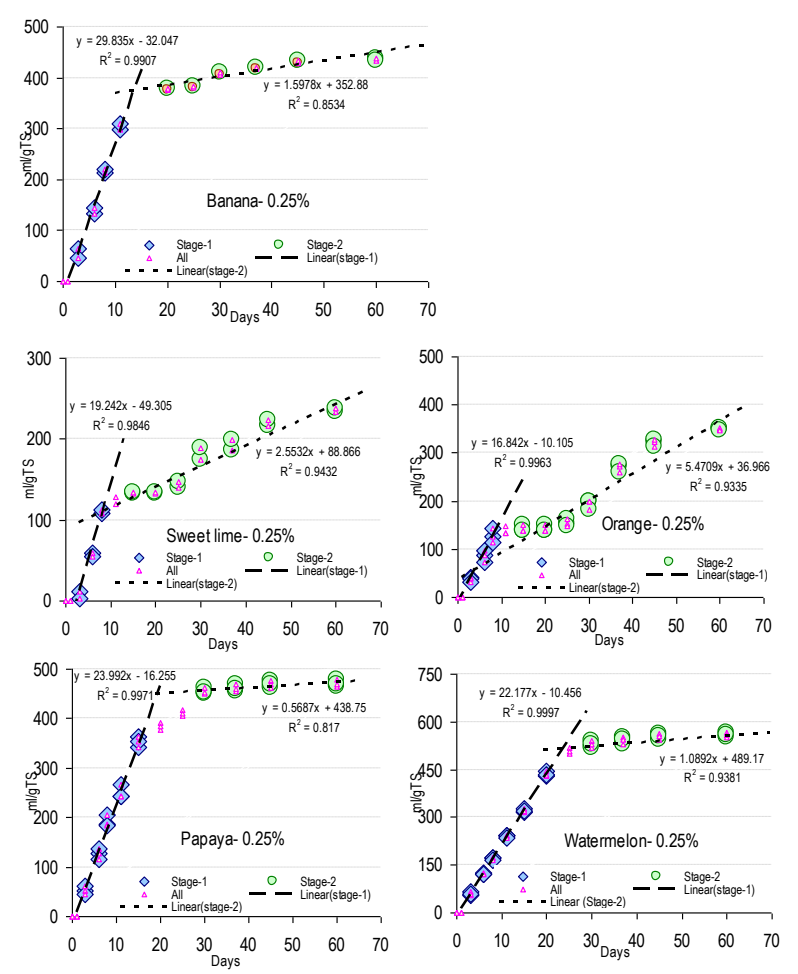

Figure 6. The cumulative gas production measured during various stages of Biological methane potential (BMP) assay for three replicates $(A, B, C)$ at $0.25 \%$ TS concentration in the fermenting mass of the commonly used five fruit feedstock (Banana, Sweet lime, Orange, Papaya, Watermelon) during phase- I and II of degradation. The black dotted line shows best linear pattern after removing outliers.

Under anaerobic decomposition, biomass feed stocks are generally reported to follow a first-order decay pattern (Smith et al., 1988; Chanakya et al., 1997a; b). MSW degradation under composting and landfill conditions is reported to follow first order kinetics (Hamoda et al., 1998; Qdais et al., 2008). The first-order rate constant was determined using gas yield patterns. Estimation of the firstorder rate constants for the two stages of decomposition using only gas production data did not give good representation of the process and is therefore not reported here. For substrates that have become dry such as onion peels, there is obviously a delay period that could range between 2 to 6 days. The gas production pattern in the BMP assay at $0.25 \%$ TS also showed a similar pattern. 

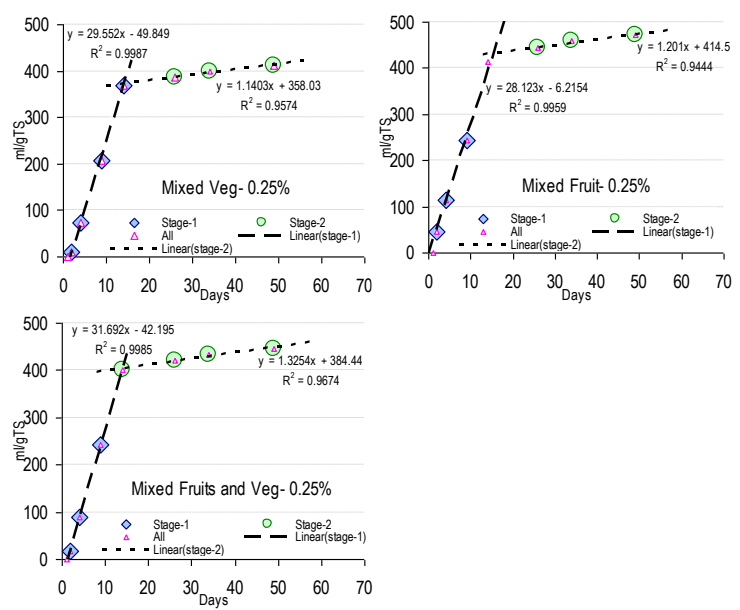

Figure 7. The cumulative gas production measured during various stages of BMP assay at $0.25 \% \mathrm{TS}$ concentration in the fermenting mass of the equal proportionally mixture of vegetables (mixed veg), fruits (mixed fruit) and mixture

of vegetables and fruits (mixed fruits and veg) during phase- I and II of degradation. The black dotted line shows best linear pattern after removing outliers.

The efficiency of conversion of feedstock to biogas, estimated as total gas production in the BMP assay as a function of theoretical gas production, is presented in Table 2. Further, the solids residence time (SRT) required for achieving the observed level of gas production (BMP) using the first stage gas production rate was estimated. Finally, the conversion efficiency measured at the level of biogas production achieved as against a theoretical potential was also estimated [Table 2; (BMP achieved per TS fed $\div$ 792) = biogas production per kg carbohydrate]. Among all the feedstocks studied, all the vegetable wastes gave a conversion efficiency of $\geq 70 \%$ (ranging from 71 $82 \%$ ). Fruit wastes (other than citrus fruit wastes) on the other hand showed a slightly lower conversion efficiency ranging from $56-78 \%$ under uninhibited conditions. Citrus fruits (sweet lime and orange peels) gave a TS-to-gas conversion efficiency ranging between $29-47 \%$ by mass. This clearly shows the presence of some form of inhibition by the presence of citrus fruit peels in the MSW. Further it may be seen that mixed fruits, mixed vegetables and mixed fruits and vegetables have a lowered conversion efficiency compared to the individual components. This ranges from $55 \%$ for mixed vegetables to about $65 \%$ for mixed fruits. This pattern while being explained by the presence of inhibitory citrus fruit peels shows a low conversion efficiency for several vegetable wastes. The potential for different mixtures to avoid the possible inhibition by citrus fruits need to be evolved in future research.

Table 2. Conversion efficiency (theoretical), solids retention time (SRT, d) and total gas production achieved during fermentation of various fruit, vegetable and mixed wastes components of MSW in the study

\begin{tabular}{ccccccc}
\hline Feedstock & \multicolumn{2}{c}{ Total Gas (BMP) } & \multicolumn{2}{c}{ SRT (d) } & \multicolumn{2}{c}{ Conv. Eff. (\%) } \\
\hline BMP @ TS in g/L & 0.5 & 0.25 & 0.5 & 0.25 & 0.5 & 0.25 \\
\hline Cauliflower & 569.6 & 548.8 & 36 & 20 & $76 \%$ & $74 \%$ \\
\hline Onion peels & 593.9 & 609.6 & 36 & 25 & $80 \%$ & $82 \%$ \\
\hline Flat beans & 604.5 & 587.7 & 30 & 15 & $81 \%$ & $79 \%$ \\
\hline Radish & 581.7 & 529.3 & 36 & 19 & $78 \%$ & $71 \%$ \\
\hline Pea shells & 582.1 & 561.6 & 29 & 16 & $78 \%$ & $75 \%$ \\
\hline Banana & 467.7 & 421.3 & 16 & 21 & $63 \%$ & $56 \%$ \\
\hline Sweet line & 215.2 & 226.0 & 11 & 20 & $29 \%$ & $30 \%$ \\
\hline Orange & 228.0 & 349.3 & 14 & 22 & $31 \%$ & $47 \%$ \\
\hline Papaya & 537.3 & 471.5 & 22 & 37 & $72 \%$ & $63 \%$ \\
\hline Water melon & 582.7 & 562.5 & 26 & 47 & $78 \%$ & $75 \%$ \\
\hline mixed vegetables & 407.0 & 413.0 & 24 & 14 & $55 \%$ & $55 \%$ \\
\hline Mixed Fruit & 484.0 & 472.0 & 27 & 17 & $65 \%$ & $63 \%$ \\
\hline Mix fruit \& vegetables & 445.6 & 446.4 & 22 & 14 & $60 \%$ & $60 \%$ \\
\hline
\end{tabular}

Total gas in $\mathrm{mL} / \mathrm{g} T S$; SRT as days for conversion at current BMP and conversion efficiency measured as per cent of gas production volume achieved as against theoretical volume at STP (STP correction not imposed)

\section{Conclusions}

MSW components at primary collection, comprising of fruit and vegetable wastes in Bangalore and many Indian cities, with very low lignin firstly suggests poor recovery in the form of compost and second rapid anaerobic degradability to VFA - all of which necessitates i. avoiding temporary storage and ii. rapid local processing. Anaerobic decomposition patterns studied here enable planning and design of decentralized processing by anaerobic composting or biomethanation. The degradation patterns can be simplified to a two stage gas production from BMP assay, where over $70 \%$ of the total BMP could be recovered in the first phase that lasts between 15 to 25 days. Among them, citrus fruits cause process problem even when used as mixtures with other fruits and vegetables and therefore alternative or augmentative processing options need to be found.

\section{Acknowledgements}

This work has been a part of a project supported by Bruhat Bengaluru Mahanagara Palike (BBMP; City Corporation), Bangalore and is gratefully acknowledged. Ms Apoorva, Ms Dhanyashree and Mr Jay K (student trainees) took up the initial work at CST. We gratefully acknowledge their help. 


\section{References}

APHA, (1975), American Health Association, Standard Methods for examination of water and wastewater, Washington DC.

Barlaz M.A., Ham R.K. and Schaefer D.M., (1989), Mass-balance analysis of anaerobically decomposed refuse, J. Environ. Eng., 115, 1088-1102.

Berg B. and McClaugherty C. (2013), Plant litter: Decomposition, humus formation, carbon sequestration, 3rd edn, Springer, Heidelberg.

Canet R. and Pomares F. (1995), Changes in physical, chemical and physicochemical parameters during the composting of municipal solid-wastes in 2 plants in Valencia, Bioresource Technology, 51(2-3), 259-264.

Cechhi F., Pavan P. and Mata-Alvarez J. (1997), Kinetic study of the thermophilic anaerobic digestion of the fresh and precomposted, mechanically selected organic fraction of municipal solid waste, J. Environ. Sci. Health, 32(1), 195-213.

Chanakya H.N., Srikumar K.G., Anand V., Modak J. and Jagdish K.S. (1997), Fermentation properties of agro-residues, leaf biomass and urban market garbage in a solid phase biogas fermenter, Biomass Bioenergy, 16, 417-429.

Chanakya H.N., Venkatsubramaniyam R. and Modak J. (1997), Fermentation and methanogenic characteristics of leafy biomass feedstocks in a solid phase biogas fermentor, Bioresource Technology, 62, 71-78.

Chanakya H.N., Srikumar K.G., Anand V., Modak J. and Jagadish K.S. (1999), Fermentation properties of agro-residues, leaf biomass and urban market garbage in a solid phase biogas fermenter, Biomass Bioenergy, 16, 417-429.

Chanakya H.N. and Sharatchandra H.C. (2005), GHG footprint of a developing country city - Bangalore, ASTRA technical report, CST Bangalore.

Chanakya H.N., Ramachandra T.V., Guruprasad M. and Devi V. (2007a), Micro-treatment options for components of organic fraction of MSW in residential areas, Environmental Monitoring and Assessment, 135, 129-139.

Chanakya H.N., Ramachandra T.V. and Vijayachamundeeswari M. (2007b), Resource recovery potential from secondary components of segregated municipal solid wastes, Environmental Monitoring and Assessment, 135, 119-127.

Chanakya H.N., Sharma I. and Ramachandra T.V. (2009a), Microscale anaerobic digestion of point source components of organic fraction of municipal solid waste, Waste Management, 29(4), 306-1312.

Chanakya H.N., Ramachandra T.V. and Shwetmala (2009b), Towards a sustainable waste management system for Bangalore, $1^{\text {st }}$ International Conference on Solid Waste Management and Exhibition on municipal services, Urban development public works, IconSWM 2009, Kolkata, India, 6367.

Chanakya H.N. and Swamy S.G.S. (2011), Status of municipal solid waste management in Karnataka - with special emphasis on small towns and cities. Technical Report Karnataka State Council for Science and Technology, Indian Institute of Science Campus, Bangalore.

Chanakya H.N., Shwetmala and Ramachandra T.V. (2011), Estimating unauthorized dumping of MSW around cities - A case study of Bangalore. 2nd International conference on sustainable waste management, ICON-SWM. 2011, Kolkata, India, 636-642.

Chanakya H.N. and Malayil S. (2012), Anaerobic retting of banana and arecanut wastes in a plug flow digester for recovery of fiber, biogas and compost, Energy for Sustainable Development, 16, 231-235.

Chanakya H.N., Shwetmala and Ramachandra T.V. (2015), Nature and extent of unauthorized dumping in and around Bangalore city, Journal of material cycles and waste management, DOI: 10.1007/s10163-015-0423-6.

Chattopadhyay S., Dutta A. and Ray S. (2009), Municipal solid waste management in Kolkata, Indian- A review, Waste management, 29, 1449-1458.

Chesson A. (1978), Maceration of linen flax under anaerobic conditions, J. Appl. Bacteriol, 45, 219-230.

Dhindaw J., Chanakya H.N. and Sitharam T.G. (2014), Neighbourhood level decentralized solid waste management facility: a prototype, Proc. Intl. Conf. on solid waste management (ICON_SWM2014), 28-30 Jan 2014, Hyderabad, India, 188-194.

Gunaseelan V.N. (2004), Biochemical methane potential of fruits and vegetable solid waste feedstocks, Biomass and Bioenergy, 26, 389-399.

Hamoda M.F., Qdais H.A. and Newham J. (1998), Evaluation of municipal solid waste composting kinetics, Resources, Conservation and Recycling, 23, 209-223.

Hill D.T. and Bolte J.P. (1989), Digester stress related to isobutyric and iso valeric acids, Biological Wastes, 28, 219-225.

Kumar N.K. and Goel S. (2009), Characterization of Municipal solid waste (MSW) and a proposed management plan for Kharagpur, West Bengal, India, Resources, conservation and recycling, 53, 166-174.

Mata-Alvarez J., Mace S. and Llabres P. (2000), Anaerobic digestion of organic solid wastes. An overview of research achievements and perspectives, Bioresource Technology, 74(1), 3-16.

Monedero Sanchez M.A., Roig A., Cegarra J. and Bernal M.P. (1999), Relationships between water-soluble carbohydrate and phenol fractions and the humification indices of different organic wastes during composting, Bioresource Technology, 70, 193-201.

NEERI, National Environmental Engineering Research Institute, (2005), Municipal solid waste Status of MSWM in 59 cities of India.

Owen J.M. and Chynoweth D.P. (1993), Biochemical methane potential of municipal solid waste (MSW) components, Water science and technologies, 27(2), 1-14.

Owen W.F., Stuckey D.C., Healy Jr J.B., Young L.Y. and McCarty P.L. (1979), Bioassay for monitoring biochemical methane potential and anaerobic toxicity, Water Research, 13, 485-492.

Pandey S.K., Tyagi P. and Gupta A.K. (2007), Municipal solid waste management in Ghazipur city- A case study, ARPN Journal of agricultural and biological science, 2(6), 41-43.

Qdais H.A. and Alsheraideh A.A. (2008), Kinetics of solid waste biodegradation in laboratory lysimeters, Jordan Journal of Civil Engineering, 2(1), 45-52.

Rajabapaiah P. (1988), Energy from Bangalore garbage- A preliminary study. ASTRA technical report, Centre for Application of Science and Technology to Rural Areas (CST), Indian Institute of Science Bangalore. 
Ravi K.D. (2014), Effect of water extractives and crude protein on the kinetics of hydrolysis in a solid state anaerobic reactor, M.Sc. Engg Thesis, Indian Institute of Science, Bangalore.

Shwetmala, Sangunni K.S., Chanakya H.N. and Harish B. (2012), Experience of involving school students in providing experiential learning and long-term participation in reducing solid wastes generation in Yelahanka New Town - Karnataka. $3^{\text {rd }}$ International Conference on Sustainable waste management - municipal, industrial and agricultural, IconSWM 2009, Mysore, India, 249-254.

Singh J.S. and Gupta S.R. (1977), Plant decomposition and soil respiration in terrestrial ecosystems, Bot. Rev, 43, 449-528.

Smith P.H., Bordeaux F.M., Goto M., Shiralipour A., Wilke A., Andrews J.F., Ide S. and Bamett M.W. (1988), Biological production of methane from biomass. In Methane from biomass, A treatment approach, Smith W.H. and Frank J.R. (Eds.) Elsevier, London, 291-334.

TIDE, Technology Informatics Design Endeavour, (2000), Energy recovery from municipal solid wastes in around Bangalore, Technical report Bangalore. 